\title{
Discovery and Characterization of an ALFA-Tag-Specific Affinity Resin Optimized for Protein Purification at Low Temperatures in Physiological Buffer
}

\author{
Markus Kilisch ${ }^{1}$, Hansjörg Götzke ${ }^{1}$ (1) , Maja Gere-Becker ${ }^{1}$, Alexander Crauel ${ }^{1}$, Felipe Opazo ${ }^{1,2,3(1)}$ \\ and Steffen Frey ${ }^{1, *(D)}$ \\ 1 NanoTag Biotechnologies GmbH, Rudolf-Wissell-Straße 28a, 37079 Göttingen, Germany; \\ markus.kilisch@nano-tag.com (M.K.); Hansjoerg.Goetzke@nano-tag.com (H.G.); \\ maja.Gere-Becker@nano-tag.com (M.G.-B.); Alexander.Crauel@nano-tag.com (A.C.); fopazo@gwdg.de (F.O.) \\ 2 Institute of Neuro- and Sensory Physiology, University Medical Center Göttingen, Humboldtallee 23, \\ 37073 Göttingen, Germany \\ 3 Center for Biostructural Imaging of Neurodegeneration (BIN), University Medical Center, \\ Von-Siebold-Straße 3a, 37075 Göttingen, Germany \\ * Correspondence: steffen.frey@nano-tag.com
}

check for

updates

Citation: Kilisch, M.; Götzke, H.; Gere-Becker, M.; Crauel, A.; Opazo, F.; Frey, S. Discovery and Characterization of an ALFA-TagSpecific Affinity Resin Optimized for Protein Purification at Low Temperatures in Physiological Buffer. Biomolecules 2021, 11, 269. https://doi.org/10.3390/ biom 11020269

Academic Editor: Nick Devoogdt

Received: 29 January 2021

Accepted: 9 February 2021

Published: 12 February 2021

Publisher's Note: MDPI stays neutral with regard to jurisdictional claims in published maps and institutional affiliations.

Copyright: (c) 2021 by the authors. Licensee MDPI, Basel, Switzerland. This article is an open access article distributed under the terms and conditions of the Creative Commons Attribution (CC BY) license (https:/ / creativecommons.org/licenses/by/ $4.0 /)$.

\begin{abstract}
Epitope tags are widely employed as tools to detect, purify and manipulate proteins in various experimental systems. We recently introduced the ALFA-tag together with two ALFA-specific single-domain antibodies (sdAbs), NbALFA and $\mathrm{NbALFA}^{\mathrm{PE}}$, featuring high or intermediate affinity, respectively. Together, the ALFA system can be employed for a broad range of applications in microscopy, cell biology and biochemistry requiring either extraordinarily stable binding or mild competitive elution at room temperature. In order to further enhance the versatility of the ALFA system, we, here, aimed at developing an sdAb optimized for efficient elution at low temperatures. To achieve this, we followed a stringent selection scheme tailored to the specific application. We found candidates combining a fast capture of ALFA-tagged proteins with an efficient competitive elution at $4{ }^{\circ} \mathrm{C}$ in physiological buffer. Importantly, by employing a structure-guided semisynthetic library based on well-characterized NbALFA variants, the high specificity and consistent binding of proteins harboring ALFA-tags at either terminus could be maintained. ALFA Selector ${ }^{C E}$, a resin presenting the cold-elutable $\mathrm{NbALFA}^{\mathrm{CE}}$, is an ideal tool for the one-step purification of sensitive protein complexes or temperature-labile enzymes. We believe that the general approach followed during the selection and screening can be transferred to other challenging sdAb discovery projects.
\end{abstract}

Keywords: nanobody; sdAbs; epitope tag; affinity; immunoprecipitation; native elution; coldelutable; synthetic library; ALFA system; ALFA Selector

\section{Introduction}

Camelid single-domain antibodies (sdAbs) [1], also referred to as nanobodies ${ }^{\circledR}$ (a trademark of Ablynx), are employed in fundamental research and clinical diagnostics, and as promising candidates for therapeutic applications. Due to their small size of $\sim 15 \mathrm{kDa}$, easy genetic accessibility and low production cost, they are ideal tools for many fields of life sciences [1-5]. Furthermore, sdAbs can be easily selected from phage-, bacterial-, or yeast-display libraries, allowing for a specific selection of binders against desired target proteins [6-8]. Although the selection of sdAbs from immune libraries generated from peripheral blood mononuclear cells (PBMCs) of immunized alpacas, llamas or camels is a straightforward process, provoking the required immune responses in these animals can be challenging. This is the case, in particular, for toxic or highly conserved proteins. In addition, immunizing animals is a time-consuming process with intrinsically unpredictable outcomes regarding the nature, quality and specificity of the sdAbs obtained. To overcome these limitations, some fully synthetic libraries have been developed, typically based on 
commonly found immunoglobulin frameworks present in camelid sdAbs [9,10]. While most sdAbs have been selected and optimized to display high affinity towards their antigens (for examples, see [11-15]), the generation of mid- and low-affinity sdAbs is of growing interest [16]. For instance, reversible binding to a specific target protein can be advantageous not only for in vivo and therapeutic use [16] but also for biochemical applications, enabling binding and elution under physiological conditions and thereby preserving the structure and function of selected target proteins [16,17].

Recently, we introduced the ALFA system, comprising the rationally designed ALFAtag and a set of highly versatile sdAbs binding ALFA-tagged proteins with extraordinary specificity regardless of the position of the tag on the protein. The high-affinity sdAb (NbALFA) shows tight binding to ALFA-tagged proteins $\left(\mathrm{K}_{\mathrm{d}} \sim 25 \mathrm{pM}\right)$ with a very slow off-rate and is ideal for applications requiring stable interactions [15]. It may, however, be suboptimal for a range of biochemical applications, as it fails to release targets under physiological conditions. In order to solve this problem, we introduced a second sdAb $\left(\mathrm{NbALFA}{ }^{\mathrm{PE}}\right.$ for "peptide-elutable") showing a strongly enhanced off-rate $\left(\mathrm{K}_{\mathrm{d}} \sim 11 \mathrm{nM}\right)$. An agarose-based resin featuring immobilized NbALFA ${ }^{\mathrm{PE}}$ (ALFA Selector ${ }^{\mathrm{PE}}$ ) allows for an efficient competitive elution of ALFA-tagged proteins under physiological conditions. In summary, the ALFA system is ideally suited for a broad spectrum of applications, including high-resolution microscopy (e.g., fluorescence microscopy, STED microscopy, DNA-PAINT, etc.) [18-20], in vivo detection and manipulation of living cells [21,22], intracellular proteins [22,23], and also advanced biochemical experimentation [15,24]. Therefore, it offers a superior and versatile alternative to most common epitope tag systems [25].

While competitive elution from ALFA Selector ${ }^{\mathrm{PE}}$ works exceptionally well in batch or stopped-flow elution protocols performed at room temperature, the elution efficiency is impaired at lower temperatures. This may limit the application of the ALFA system, especially for the purification of temperature-sensitive targets or delicate protein complexes, or in the case that it is essential to perfectly preserve structure and function. Therefore, we set out to complement the ALFA system by adding a third sdAb that allows for the efficient capture and elution of proteins at $4{ }^{\circ} \mathrm{C}$ and under physiological buffer conditions while maintaining the exquisite specificity and favorable biochemical properties of the existing NbALFA variants [15]. To achieve this, we combined a structure-guided protein engineering approach with a novel off-rate-driven phage display selection. We demonstrate that the applied rational design principles in combination with an application-specific selection protocol can lead to affinity reagents fulfilling the experimental requirements in a directed fashion. We finally present $\operatorname{NbALFA}^{\mathrm{CE}}$ (for "cold-elutable"), a new member of the ALFA system, which proves to be an ideal tool for the purification and elution of ALFA-tagged target proteins at cold temperatures in physiological buffer.

\section{Materials and Methods}

\subsection{Animal Handling - Immunizations}

All the work involving animal experiments was conducted in compliance with ethical regulations for animal research and testing. All experiments conducted did not require ethical approval, but were communicated to and accepted by the local authorities (LAVES, Niedersachsen, Germany). Two alpacas were immunized six times at 14-day intervals with a total of $0.5 \mathrm{mg}$ of ALFA peptide conjugated to keyhole limpet hemocyanin (KLH). The first immunization was performed using complete Freund's adjuvant; for all the following immunizations, incomplete Freund's adjuvant was used. Five days after the last immunization, $100 \mathrm{~mL}$ of peripheral blood was taken from each animal and immediately supplemented with $5000 \mathrm{IU} / \mathrm{mL}$ of heparin to prevent clotting.

\subsection{Preparation of Phagemid Libraries}

Two independent phagemid libraries were constructed for the selection of ALFAspecific binders that can be peptide-eluted at $4{ }^{\circ} \mathrm{C}$. The first library was prepared from total ribonucleic acid (RNA) isolated from peripheral blood mononuclear cells (PBMCs) 
obtained from fresh alpaca blood. The PBMCs were isolated using Ficoll-Paque PLUS (GE Healthcare, Uppsala, Sweden). Subsequently, total RNA was isolated using a NucleoSpin RNA plus kit (Macherey Nagel, Düren, Germany). The obtained RNA was used for a reverse transcription reaction using Superscript IV Reverse Transcriptase (Thermo Fisher Scientific, Waltham, MA, USA). SdAb-encoding sequences were amplified by a two-step nested polymerase chain reaction (PCR) using the primers CaLl 01 and CaLl 02 [26] and primers F1 and R1 (Table S1) in a second PCR reaction. The final PCR product was cloned into a pHen2-derived phagemid vector and transformed into TG1 cells, yielding an sdAb library with a complexity of $\sim 2 \times 10^{8}$ individual clones.

For the preparation of the second sdAb library, a structure-guided approach was chosen. Based on the previously published structure of NbALFA bound to an ALFA peptide ([15], PDB: 6I2G), various mutations were introduced to lower the binding affinity of $\mathrm{NbALFA}$ and $\mathrm{NbALFA}^{\mathrm{PE}}$ towards their substrate while ideally retaining their binding specificity. For that, the respective coding sequences were cloned into a pHen2-derived phagemid vector. Then, in both templates, cysteine residue 24 (numbering according to PDB: 6I2G) was mutated to serine. In all four plasmids, all CDR3 residues forming direct contacts with the ALFA peptide were randomized by saturation mutagenesis using degenerate NNK codons, resulting in a library with a theoretical complexity of $6.4 \times 10^{5}$ individual sdAbs. All mutagenesis steps were carried out using primer-directed PCR mutagenesis. The combined pool of mutant phagemids was transformed into TG1 cells, yielding $\sim 1.2 \times 10^{9}$ individual clones.

\subsection{Selection of Binders-Biopanning}

For each library, three subsequent biopanning steps were performed (Figure 1C-E). In each biopanning step, the experimental conditions were adjusted and the stringency, increased. For the first biopanning step, $100 \mu \mathrm{L}$ of streptavidin-coated MyOne DynaBeads (Thermo Fisher Scientific, Waltham, MA, USA) were loaded with a total of $200 \mathrm{pmol}$ biotinylated target protein (shGFP2-ALFA). The mixture was incubated for $1 \mathrm{~h}$ at room temperature, washed three times with $1 \mathrm{~mL}$ of phosphate-buffered saline (PBS) and added to a phage suspension containing $2.0 \times 10^{12}$ phages pre-blocked with $20 \mu \mathrm{g} / \mathrm{mL}$ bovine serum albumin (BSA). The suspension was incubated for $1 \mathrm{~h}$ at room temperature, transferred to a $4{ }^{\circ} \mathrm{C}$ environment and washed once at $4{ }^{\circ} \mathrm{C}$ for $30 \mathrm{~min}$ with $10 \mathrm{~mL}$ of PBS. The remaining binders were eluted at $4{ }^{\circ} \mathrm{C}$ with $0.5 \mathrm{~mL}$ of PBS containing a 1000-fold excess of ALFA peptide ( $200 \mathrm{pmol}$ target protein, $200 \mathrm{nmol}$ ALFA peptide). Eluted phages were used to infect $E$. coli TG1 cells for $1 \mathrm{~h}$ at $37^{\circ} \mathrm{C}$. The infected TG1 cells were grown overnight at $37^{\circ} \mathrm{C}$. The next day, the culture was diluted and grown to an optical density (OD) of 0.6 before infection with a MK13KO7 helper phage for $1 \mathrm{~h}$ at $37^{\circ} \mathrm{C}$. The phages were selected by adding kanamycin $(50 \mu \mathrm{g} / \mathrm{mL})$ and grown overnight at $30^{\circ} \mathrm{C}$. The next day, the phages were purified from the cleared culture supernatants by repeated precipitation with 5\% PEG-8000 and $1.5 \mathrm{M} \mathrm{NaCl}$ (final concentrations in suspension: 1\% PEG-8000 and $300 \mathrm{mM} \mathrm{NaCl}$ ) and resuspended in a final volume of $4 \mathrm{~mL}$ of resuspension buffer. The second biopanning step was performed in a similar way while reducing the amount of target protein (ALFA-biotin) immobilized on the beads to $20 \mathrm{pmol}$ and the elution time to $15 \mathrm{~min}$ at $4{ }^{\circ} \mathrm{C}$. For the last biopanning step, 20 pmol of biotin-ALFA was used as a bait. The elution time was reduced to $5 \mathrm{~min}$ at $4{ }^{\circ} \mathrm{C}$.

After three rounds of selection, the obtained sdAb-containing libraries were cloned into a pQE-derived expression vector. A total of 96 single clones per library were expressed. Crude lysates were prepared and tested by enzyme-linked immunosorbent assay (ELISA) for their binding properties and their ability to be peptide-eluted at $4{ }^{\circ} \mathrm{C}$. Positive clones were sequenced, aligned and grouped into families. Representative clones were further analyzed biochemically. 


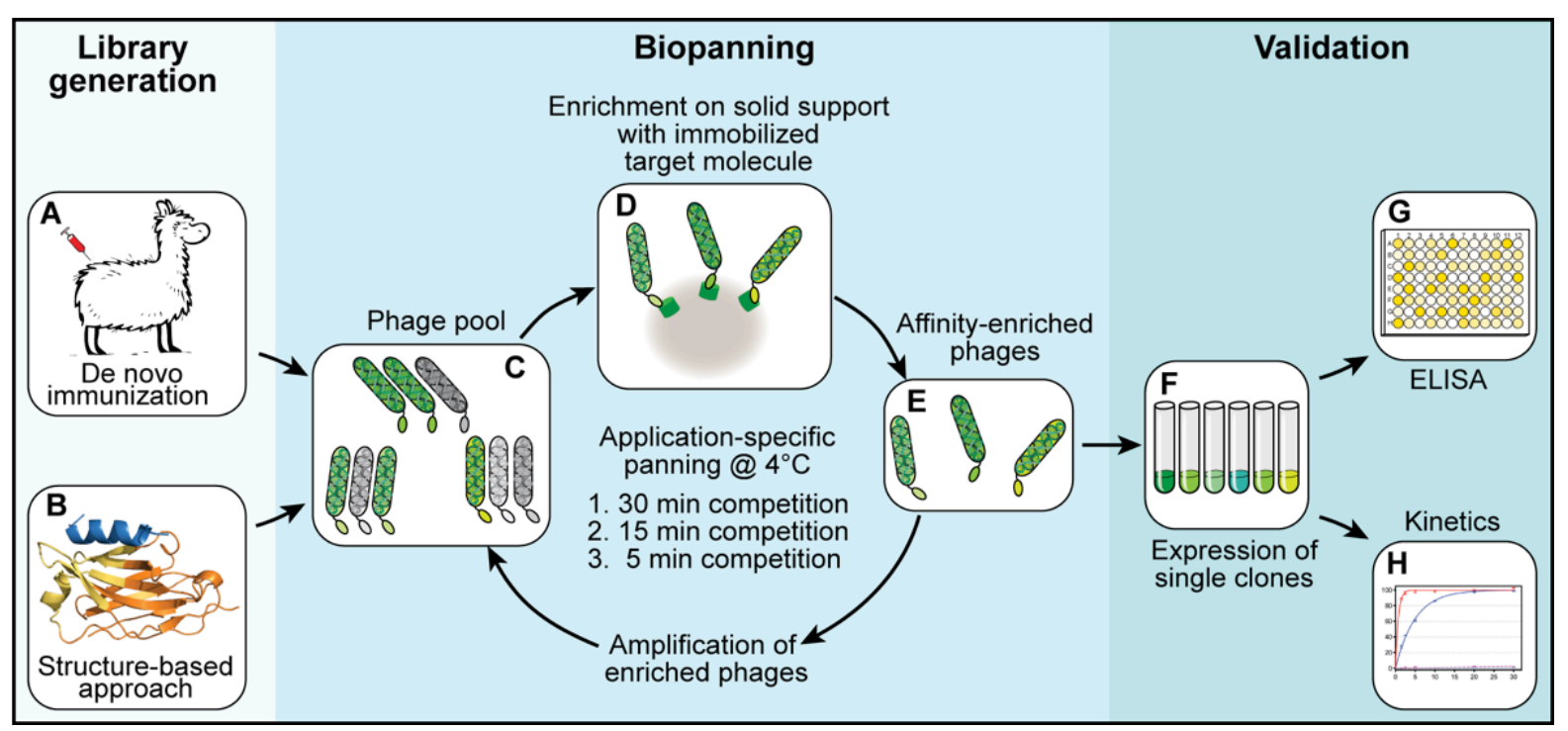

Figure 1. Schematic depiction of the workflow followed to create cold-elutable ALFA binders. Two separate sdAb libraries were generated by either de novo immunization of alpacas (A) or a structure-guided approach based on the known structure of NbALFA in complex with the ALFA peptide (B). The resulting phage pools were separately used as input material for phage displays (C-E). In three rounds of biopanning, the selection stringency was increased by reducing both the amount of immobilized target protein and the time allowed for competitive elution at $4{ }^{\circ} \mathrm{C}$. Eluted phages were amplified and used as input for the next round of biopanning. After three rounds of biopanning, enriched NbALFA ${ }^{\mathrm{CE}}$ candidates were subcloned and expressed in E. coli (F). Candidates were further assayed by ELISA for their binding and elution properties (G) or subjected to a kinetic characterization $(\mathbf{H})$.

\subsection{Protein Expression and Purification}

Recombinant proteins were expressed in E. coli from pQE-derived expression vectors (Table S2). The recombinant proteins ALFA-shGFP2, shGFP2-ALFA, NbALFA, NbALFA ${ }^{\mathrm{PE}}$ and $\mathrm{NbALFA}{ }^{\mathrm{CE}}$ were expressed as N-terminal His 14 -bdSUMO fusion proteins. In general, E. coli transformed with the respective plasmids were cultured in terrific broth (TB) medium until an OD of 4.0 was reached. Protein expression was induced by the addition of $0.3 \mathrm{mM}$ isopropyl thiogalactopyranoside (IPTG) at $23^{\circ} \mathrm{C}$, overnight. Before the cells were harvested by centrifugation, the cultures were supplemented with $5 \mathrm{mM}$ ethyleneimine tetraacetate (EDTA) and $1 \mathrm{mM}$ phenylmethylsulfonyl fluoride (PMSF). Subsequently, the cells were lysed in LS buffer (50 mM Tris/ $\mathrm{HCl}, \mathrm{pH} 7.5 ; 300 \mathrm{mM} \mathrm{NaCl} ; 5 \mathrm{mM}$ EDTA) supplemented with $15 \mathrm{mM}$ imidazole/ $\mathrm{HCl}, \mathrm{pH} 7.5$, and $10 \mathrm{mM}$ dithiothreitol (DTT). The crude lysates were cleared of remaining cellular debris and bound to $\mathrm{Ni}^{2+}$-chelate beads for $1 \mathrm{~h}$ at $4{ }^{\circ} \mathrm{C}$. The protein-loaded beads were washed extensively, and the proteins were cleaved on column with $100 \mathrm{nM}$ bdSENP1 [27] for $1 \mathrm{~h}$ at $4{ }^{\circ} \mathrm{C}$. All proteins were subsequently subjected to size exclusion chromatography. Coupling to an $\mathrm{SH}$-reactive, agarose-based resin was performed using an ectopic cysteine fused to the C-terminus of the respective $\mathrm{NbALFA}$ variants via a hydrophilic linker. Product numbers of the resulting Selector resins are summarized in Table S3.

\subsection{Off-Rate Assays}

For an estimation of the off-rates for ALFA Selector ${ }^{\mathrm{PE}}$ and analogous resins coupled to NbALFA ${ }^{\mathrm{CE}}$ candidates, $20 \mu \mathrm{L}$ of substrate-saturated resin was washed four times with PBS and resuspended in $200 \mu \mathrm{L}$ of PBS containing $200 \mu \mathrm{M}$ ALFA peptide. At given time points, the progression of elution was quantified by measuring the fluorescence of the GFP-tagged protein released into the supernatant (QBit 3.0; Thermo-Fischer Scientific, Waltham, MA, USA). After the kinetic measurements, all reactions were adjusted to $200 \mu \mathrm{M}$ peptide concentrations and incubated for $30 \mathrm{~min}$ at $30^{\circ} \mathrm{C}$. The fluorescence values obtained after such post-elution were set to $100 \%$. For the experiments shown in Figure 2E,F, each 
data point represents the average of four independent experiments performed in parallel. The statistical analyses and curve fittings were performed using GraphPad Prism 5.0.

A

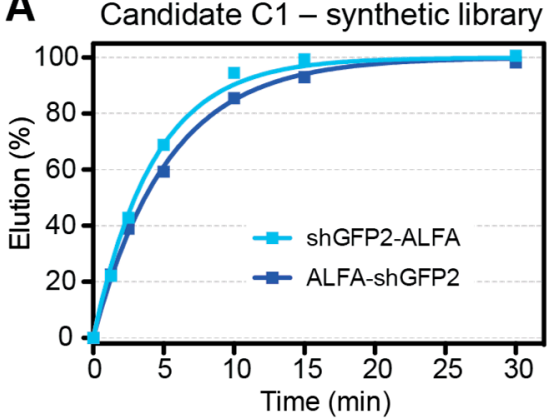

C Candidate A12-immune library

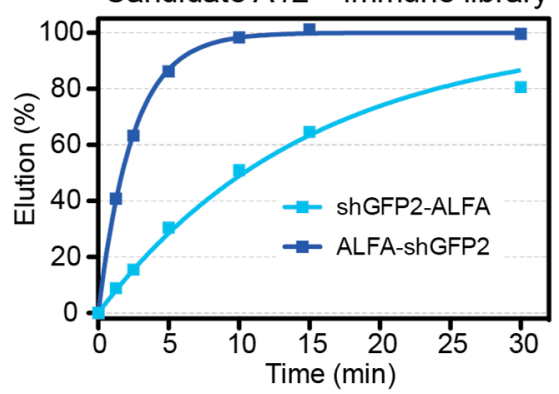

E

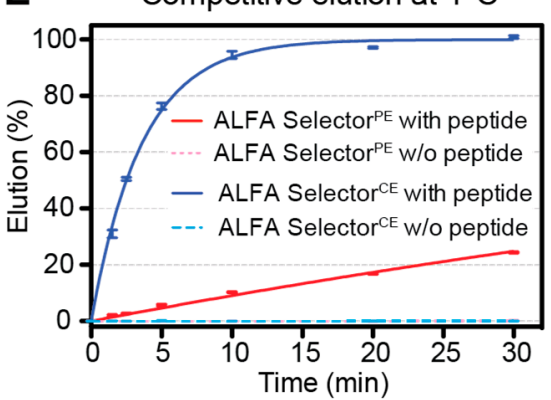

B Candidate $\mathrm{E} 10$ - synthetic library

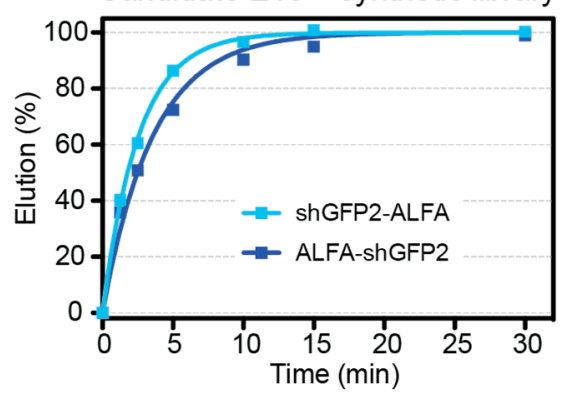

D Candidate F10 - immune library

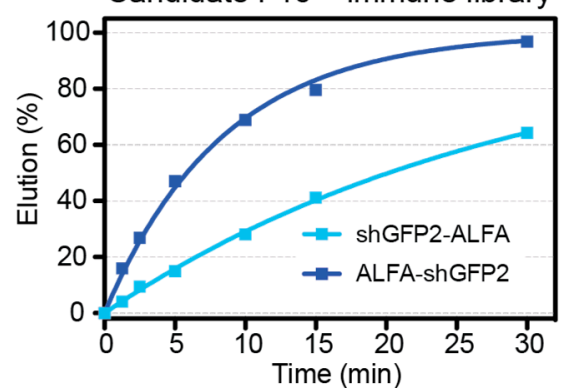

F $\quad$ Competitive elution at $25^{\circ} \mathrm{C}$

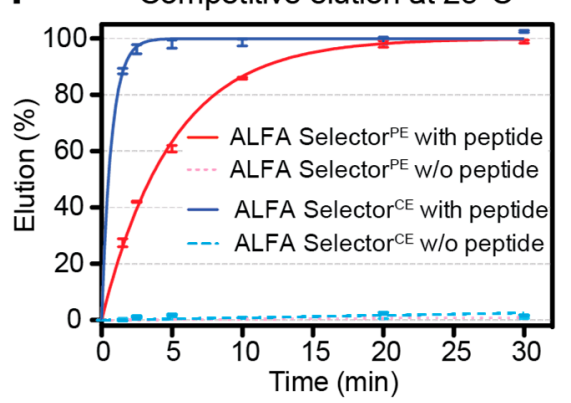

Figure 2. Kinetic characterization of $\mathrm{NbALFA}^{\mathrm{CE}}$ candidate clones. (A-D): Selected NbALFA ${ }^{\mathrm{CE}}$ candidates were immobilized on an agarose-based resin. All resins were charged with either shGFP2ALFA (light blue curves) or ALFA-shGFP2 (dark blue curves). Upon competitive elution in batch mode using $200 \mu \mathrm{M}$ ALFA peptide at $4{ }^{\circ} \mathrm{C}$, target protein elution was quantified by measuring the fluorescent material released into the supernatant. (E,F): ALFA Selector ${ }^{\mathrm{PE}}$ (PE, red curves) and ALFA Selector ${ }^{\mathrm{CE}}$ based on candidate clone $\mathrm{C} 1$ (CE, blue curves) were charged with shGFP2-ALFA and subjected to competitive peptide elution at $4{ }^{\circ} \mathrm{C}(\mathrm{E})$ or $25^{\circ} \mathrm{C}(\mathrm{F})$, respectively. Parallel reactions without addition of peptide served as controls (dotted red and dotted blue curves). Elution was quantified as described for panels (A-D). The data points show mean values from four independent experiments including standard deviations. Curves represent fits to first-order association kinetics.

\subsection{Titration of ALFA Peptide}

For each experiment, $250 \mu \mathrm{L}$ of ALFA Selector ${ }^{\mathrm{CE}}$ was saturated with shGFP2-ALFA, extensively washed with PBS containing 0.02\% Triton X-100 (TX-100) and packed in a $1 \mathrm{~mL}$ syringe equipped with a porous bottom filter frit. For stopped-flow elution at 22 or $4{ }^{\circ} \mathrm{C}$, $70 \mu \mathrm{L}$ aliquots of PBS $+0.02 \%$ TX-100 containing various concentrations of ALFA peptide were added either every $1.5 \mathrm{~min}$ (effective flow rate, 0.18 column volumes $(\mathrm{CV}) / \mathrm{min}$ ) or every $5 \mathrm{~min}$ (effective flow rate, $0.056 \mathrm{CV} / \mathrm{min}$ ) and allowed to enter the column by gravity flow while collecting the eluate. In each fraction, the released protein was quantified fluorometrically (QBit 3.0; Thermo-Fischer Scientific, Waltham, MA, USA). 


\subsection{Affinity Purification from E. coli and HeLa Lysates}

Affinity-purification experiments with E. coli or HeLa lysates were performed with a defined amount of ALFA-tagged target protein. Therefore, cleared E. coli lysate or HeLa S100 lysate was blended with $3 \mu \mathrm{M}$ purified ALFA-tagged target protein (ALFA-shGFP2, shGFP2-ALFA). For each experiment, $25 \mu \mathrm{L}$ of the indicated ALFA Selector ${ }^{\mathrm{ST}} / \mathrm{PE} / \mathrm{CE}$ (NanoTag Biotechnologies, Göttingen, Germany, Cat No. N1511, N1510, N1512) was incubated with $1 \mathrm{~mL}$ of lysate containing the indicated ALFA-tagged target protein for $1 \mathrm{~h}$ at room temperature or at $4{ }^{\circ} \mathrm{C}$. After binding, the resins were washed three times in batch with $1 \mathrm{~mL}$ of PBS, transferred to a MiniSpin column and washed twice with $0.6 \mathrm{~mL}$ of PBS. The resin was then resuspended in $50 \mu \mathrm{L}$ of PBS containing $200 \mu \mathrm{M}$ ALFA peptide and incubated for $15 \mathrm{~min}$ at room temperature or at $4{ }^{\circ} \mathrm{C}$. All Selector resins were additionally incubated in SDS sample buffer to remove the remaining proteins, heating the samples to $95^{\circ} \mathrm{C}$ for $5 \mathrm{~min}$. As specificity controls, the indicated ALFA Selectors were incubated with E. coli or HeLa lysate lacking any ALFA-tagged target protein. The samples were resolved by SDS-PAGE and analyzed by Coomassie staining.

\subsection{Affinity Purification of Low-Abundant Proteins from HeLa Lysates}

A volume of $50 \mathrm{~mL}$ of HeLa S100 extract was blended with $100 \mathrm{nM}$ shGFP2-ALFA and applied to $1 \mathrm{~mL}$ of ALFA Selector ${ }^{\mathrm{CE}}$ resin (NanoTag Biotechnologies, Göttingen, Germany, Cat No. N1512) at a flow rate of $0.8-1.0 \mathrm{~mL} / \mathrm{min}$. The resin was washed with $10 \mathrm{CV}$ of PBS and eluted by a stepwise addition of PBS containing $1 \mathrm{mM}$ ALFA peptide $(250 \mu \mathrm{L}$ every $3 \mathrm{~min}$ ) at room temperature. Eluate fractions containing the target protein were pooled. All samples were resolved by SDS-PAGE and further analyzed by Western Blotting. The ALFA-tagged protein was detected using an ALFA-specific HRP-coupled sdAb (NanoTag Biotechnologies, Göttingen, Germany, Cat No. N1501-HRP; Table S3). Western blotting was essentially performed as described before by Götzke et al. in 2019 [15].

\subsection{Resistance to Stringent Washing and $\mathrm{pH}$}

A volume of $20 \mu \mathrm{L}$ of ALFA Selector ${ }^{\mathrm{PE}}$ or ALFA Selector ${ }^{\mathrm{CE}}$ was saturated with either ALFA-shGFP2 or shGFP2-ALFA. The beads were washed four times with Tris-buffered saline (TBS: $25 \mathrm{mM}$ Tris/ $\mathrm{HCl}, \mathrm{pH} 7.5 ; 150 \mathrm{mM} \mathrm{NaCl} ; 2 \mathrm{mM}$ EDTA) and subsequently incubated in $200 \mu \mathrm{L}$ of the indicated solutions or buffers for $2 \mathrm{~h}$ at room temperature with shaking. For post-elution, $250 \mu \mathrm{M}$ ALFA peptide was added, and the reactions were further incubated for $30 \mathrm{~min}$ at $25^{\circ} \mathrm{C}$. The eluates were quantified using a fluorometer (QBit 3.0; Thermo-Fischer Scientific, Waltham, MA, USA) before and after post-elution with ALFA peptide. Pictures were taken upon UV illumination using a Nikon D700 camera equipped with a $105 \mathrm{~mm}$ macro lens (Nikon, Minato, Japan).

\subsection{Regeneration of ALFA Selector $C E$}

Regeneration experiments were performed using an Äkta FPLC (Amersham Biosciences, Little Chalfont, UK). A $0.5 \mathrm{~mL}$ volume of ALFA Selector ${ }^{\mathrm{CE}}$ was packed in a glass column and equilibrated with PBS before saturating it with ALFA-shGFP2. The loaded columns were washed with $10 \mathrm{CV}$ of PBS, and the proteins were eluted in flow $(0.1 \mathrm{~mL} / \mathrm{min})$ with PBS containing $1 \mathrm{mM}$ ALFA peptide (elution buffer). Eluate fractions containing the target protein were pooled. The protein content was quantified by measuring the absorbance at $280 \mathrm{~nm}$ and additionally analyzed by SDS-PAGE. The column was regenerated with $10 \mathrm{CV}$ of glycine $\mathrm{pH} 2.2(100 \mathrm{mM}$ glycine, $\mathrm{pH} 2.2 ; 150 \mathrm{mM} \mathrm{NaCl})$ and re-equilibrated with $10 \mathrm{CV}$ of PBS. After the first regeneration, binding of ALFA-shGFP2 and elution were repeated as described above. The resin was subjected to nine additional regeneration cycles with glycine, before the final target protein loading and elution was performed. All samples were resolved by SDS-PAGE and visualized by Coomassie staining. The experiment was repeated with fresh resin using $100 \mathrm{mM} \mathrm{NaOH}$ instead of glycine for regeneration. The elution profiles were plotted using GraphPad Prism 5.0. 


\section{Results}

\subsection{Selection of Cold-elutable ALFA Binders}

The primary aim of this study was to develop an sdAb that would be able to specifically and efficiently capture ALFA-tagged target proteins while being suitable for an efficient competitive elution at $4{ }^{\circ} \mathrm{C}$. We started by creating two independent sdAb libraries (Figure 1). The first sdAb library was obtained from alpacas immunized with ALFA peptides (Figure 1A). The second, semisynthetic sdAb library was constructed using a structure-guided approach (Figure 1B). Here, based on the known structure of substratebound NbALFA (PDB: 6I2G), multiple positions within the CDR and scaffold regions of $\mathrm{NbALFA}$ were either randomized or mutated (see Materials and Methods). The resulting $\mathrm{sdAb}$ libraries were screened by a customized, off-rate-driven phage display using cycles of binding, stringent washing and competitive peptide elution at $4{ }^{\circ} \mathrm{C}$ (Figure 1C-E). The selection stringency was successively increased by reducing the time allowed for competitive phage elution from an initial $30 \mathrm{~min}$ to only $5 \mathrm{~min}$ in cycle 3 . Using this approach, we ensured an enrichment of sdAbs that stably associated with ALFA-tagged proteins while still allowing for an efficient release at $4{ }^{\circ} \mathrm{C}$. After three panning cycles, the NbALFA ${ }^{\mathrm{CE}}$ candidates were cloned into a prokaryotic expression vector, expressed in E. coli (Figure 1F) and analyzed by ELISA for the desired properties (Figure 1G,H). From the 192 clones analyzed, nine nonredundant $\mathrm{NbALFA}{ }^{\mathrm{CE}}$ candidates representing the most dominant $\mathrm{sdAb}$ families were chosen for further characterization.

\subsection{Novel ALFA Binders Facilitate Competitive Elution of ALFA-Tagged Target Proteins at $4{ }^{\circ} \mathrm{C}$}

All selected $\mathrm{NbALFA}{ }^{\mathrm{CE}}$ candidates were recombinantly expressed in E. coli, purified and immobilized on an agarose-based resin. For an initial characterization, each resin was loaded with a green fluorescent protein variant (shGFP2) [28] fused to a single ALFA-tag (shGFP2-ALFA) and used as a visible model substrate to follow elution on ice upon addition of an excess of ALFA peptide. The binding and the elution kinetics were semiquantitatively assessed by visual inspection (Table 1 ).

Table 1. Binding properties of selected ALFA binders obtained from an immune library and a semisynthetic library.

\begin{tabular}{|c|c|c|c|c|c|c|}
\hline \multicolumn{2}{|c|}{ NbALFA $^{\text {CE }}$ Candidate on Resin } & \multicolumn{5}{|c|}{ Remaining Signal after Competitive Elution on Ice } \\
\hline Clone & Origin of Library & $0 \mathrm{~min}$ & $2 \mathrm{~min}$ & $5 \mathrm{~min}$ & $10 \mathrm{~min}$ & $30 \mathrm{~min}$ \\
\hline $\mathrm{C} 1$ & Semisynthetic & +++ & + & $(+)$ & - & - \\
\hline D3 & Semisynthetic & +++ & $(+)$ & - & - & - \\
\hline E5 & Semisynthetic & +++ & $(+)$ & - & - & - \\
\hline E10 & Semisynthetic & +++ & ++ & + & $(+)$ & - \\
\hline H11 & Semisynthetic & +++ & $(+)$ & - & - & - \\
\hline A12 & Immune library & +++ & + & $(+)$ & - & - \\
\hline C6 & Immune library & +++ & + & - & - & - \\
\hline F9 & Immune library & +++ & ++ & + & $(+)$ & $(+)$ \\
\hline F10 & Immune library & +++ & ++ & + & $(+)$ & - \\
\hline \multicolumn{2}{|c|}{$\mathrm{NbALFA}^{\mathrm{PE}}$ control } & +++ & +++ & +++ & ++ & + \\
\hline
\end{tabular}

Legend: +++: target protein completely bound to Selector resin; ++: moderate decrease in signal after competitive elution; +: strong decrease in signal after competitive elution; $(+)$ : barely measurable signal after competitive elution; -: no signal remaining on resin.

While all the candidates were able to capture shGFP2-ALFA, the observed dissociation kinetics differed considerably. When compared to $\mathrm{NbALFA}^{\mathrm{PE}}$, which was used as a control and is known to only slowly release target proteins at low temperature, the elution from all $\mathrm{NbALFA}{ }^{\mathrm{CE}}$ candidate clones was much more efficient. The $\mathrm{NbALFA}^{\mathrm{CE}}$ candidates $\mathrm{C} 1$, 
E10, A12 and F10 showed efficient elution of shGFP2-ALFA within an optimal timeframe of 5-10 min and were selected for a more detailed analysis. Clones with slower or faster elution kinetics were excluded from all further analyses.

For a more thorough characterization, agarose resins coupled to the $\mathrm{NbALFA} C E$ candidates C1, E10, A12 and F10 were loaded with shGFP2 substrates harboring an N- or C-terminal ALFA-tag, respectively. After washing, ALFA peptide was added in excess, and the elution kinetics at $4{ }^{\circ} \mathrm{C}$ were followed by measuring the fluorescent material released into the supernatant (Figure 2). The amount of ALFA peptide used in this "batch elution mode" (200 $\mu \mathrm{M}$ peptide and 10 resin bed volumes) corresponded to an $\sim 15$-fold excess over the available binding sites, which is sufficient to trigger an efficient competitive elution. For all the resins addressed, the elution kinetics of shGFP2-ALFA were consistent with the rough evaluations conducted before (Table 1). However, striking differences were observed when comparing the elution of shGFP2 harboring N- or C-terminal ALFA-tags: both resins coupled to the $\mathrm{NbALFA}{ }^{\mathrm{CE}}$ candidates $\mathrm{C} 1$ and E10 originating from the semisynthetic library showed very similar and consistent elution kinetics for substrates tagged at either terminus (Figure 2A,B). By contrast, the elution kinetics of the two substrates differed strikingly when analyzing the resins coupled to the $\mathrm{NbALFA}^{\mathrm{CE}}$ candidates originating from the immune library (Figure $2 \mathrm{C}, \mathrm{D}$ ). As an $\mathrm{NbALFA}^{\mathrm{CE}}$ candidate should ideally recognize ALFA-tagged proteins irrespective of the localization of the ALFA-tag within the target protein, we excluded both $\mathrm{NbALFA}^{\mathrm{CE}}$ candidates originating from the immune library. Due to its slightly stronger binding to both ALFA-tagged substrates, we finally selected the candidate $\mathrm{C} 1$ as our final $\mathrm{NbALFA}{ }^{\mathrm{CE}}$. This clone combines a robust binding of ALFAtagged substrates with a sufficiently fast and consistent elution kinetics at $4{ }^{\circ} \mathrm{C}$ irrespective of the localization of the ALFA-tag within the target protein. The agarose-based resin featuring immobilized $\mathrm{NbALFA}{ }^{\mathrm{CE}}$ was called ALFA Selector ${ }^{\mathrm{CE}}$.

When directly comparing the elution kinetics of ALFA Selector ${ }^{\mathrm{PE}}$ and ALFA Selector ${ }^{\mathrm{CE}}$ at 4 and $25^{\circ} \mathrm{C}$, major differences could be observed (Figure 2E,F). While competitive elution was possible within $15-20 \mathrm{~min}$ at $4^{\circ} \mathrm{C}$ or $3 \mathrm{~min}$ at $25^{\circ} \mathrm{C}$ when using ALFA Selector ${ }^{\mathrm{CE}}$, efficient elution from ALFA Selector ${ }^{\mathrm{PE}}$ could only be achieved at $25^{\circ} \mathrm{C}$. For both resins, any spontaneous leakage of bound target protein in the absence of peptide was negligible.

\subsection{Impact of ALFA Peptide Concentration on Elution}

Having established that ALFA-tagged proteins can be eluted efficiently from ALFA Selector ${ }^{\mathrm{CE}}$ at $4{ }^{\circ} \mathrm{C}$, the impact of the ALFA peptide concentration on the elution of an ALFA-tagged target protein was tested. In a first set of experiments, ALFA Selector ${ }^{C E}$ saturated with shGFP2-ALFA was subjected to competitive elution with varying ALFA peptide concentrations at $4{ }^{\circ} \mathrm{C}$ and room temperature $\left(\sim 22^{\circ} \mathrm{C}\right)$ in a "stopped-flow" setup (Figure S1A,B). In this elution mode, the elution buffer is added intermittently in small portions, resulting in a low net flow rate. In a third experiment, competitive elution was performed at $22{ }^{\circ} \mathrm{C}$ in "gravity-flow" mode, resulting in a higher net flow rate. Under all the conditions tested, the peak protein concentration and the sharpness of the elution peak increased upon raising the peptide concentration. When using $1 \mathrm{mM}$ ALFA peptide during elution, $>90-95 \%$ of the target protein could be recovered within 3-4 fractions (0.84-1.12 column volumes), indicating that efficient elution is possible at $4{ }^{\circ} \mathrm{C}$ in "stoppedflow" mode (Figure S1A) and at $22^{\circ} \mathrm{C}$ even in flow mode (Figure S1C). Even higher peptide concentrations led to only marginally better results.

\subsection{Affinity Purification Using Different ALFA Selector Variants}

We next wanted to compare the features of the well-characterized high-affinity ALFA Selector $^{\mathrm{ST}}$ and intermediate-affinity ALFA Selector ${ }^{\mathrm{PE}}$ [15] with the novel ALFA Selector ${ }^{\mathrm{CE}}$ in single-step affinity purification experiments using shGFP2-ALFA at 22 and $4{ }^{\circ} \mathrm{C}$ (Figure 3A,B). As expected, all the resins specifically bound the ALFA-tagged target protein at both temperatures with comparable capacity. Consistent with our previous observations [15], the competitive peptide elution from ALFA Selector ${ }^{\mathrm{ST}}$ was generally 
inefficient at all the tested temperatures. Thus, the bound proteins had to be post-eluted with SDS sample buffer (Figure 3A,B, left panel). By contrast, the bound target protein could be efficiently released by peptide elution under native conditions at $22{ }^{\circ} \mathrm{C}$ from both ALFA Selector ${ }^{\mathrm{PE}}$ and ALFA Selector ${ }^{\mathrm{CE}}$ (Figure $3 \mathrm{~A}, \mathrm{~B}$, middle and right panels). When comparing the efficiency of peptide elution at $4{ }^{\circ} \mathrm{C}$, differences became apparent between ALFA Selector ${ }^{\mathrm{PE}}$ and ALFA Selector ${ }^{\mathrm{CE}}$ : while the peptide elution from ALFA Selector ${ }^{\mathrm{PE}}$ was inefficient, the target protein was readily released from ALFA Selector ${ }^{\mathrm{CE}}$ under identical conditions (Figure 3B, middle and right panels).

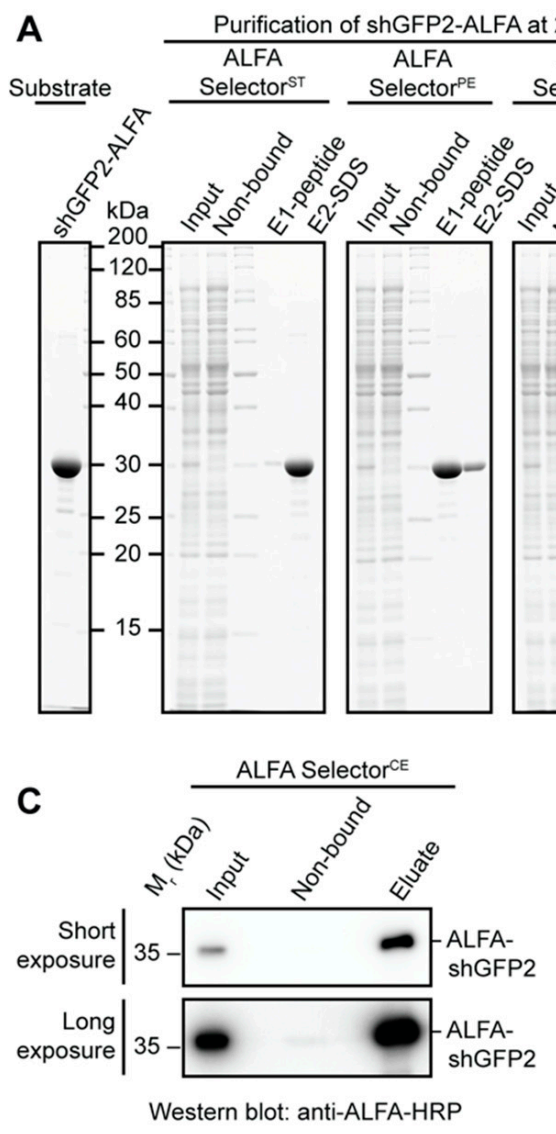

Figure 3. Affinity purification of ALFA-tagged proteins from complex lysates. (A,B): An E. coli lysate was blended with $3 \mu \mathrm{M}$ recombinant purified shGFP2-ALFA $(\mathbf{A}, \mathbf{B})$ and incubated with ALFA Selector ${ }^{\mathrm{ST}}$, ALFA Selector ${ }^{\mathrm{PE}}$ or ALFA Selector ${ }^{\mathrm{CE}}$. After washing, charged resins were eluted with $200 \mu \mathrm{M}$ ALFA peptide at $22{ }^{\circ} \mathrm{C}(\mathrm{A})$ or $4{ }^{\circ} \mathrm{C}$ (B) (E1-peptide). For all Selectors, remaining proteins were subsequently eluted with hot SDS sample buffer (E2-SDS). Amounts loaded in the SDS-PAGE for all eluate fractions correspond to the material eluted from $1 \mu \mathrm{L}$ of Selector resin. Images show gels after staining with Coomassie Brilliant Blue. (C): One-step affinity purification of low-abundant proteins using ALFA Selector ${ }^{\mathrm{CE}}$ : $50 \mathrm{~mL}$ of HeLa lysate containing $100 \mathrm{nM}$ ALFA-shGFP2 was passed over $1 \mathrm{~mL}$ of ALFA Selector ${ }^{\mathrm{CE}}$ at room temperature using gravity flow. After washing, the column was eluted with PBS containing $1 \mathrm{mM}$ ALFA peptide. Fractions were pooled and analyzed by SDS-PAGE and Western blotting. Amounts loaded correspond to 1/20,000 of the input and flow-through material and $1 / 2000$ of the eluate. Shown is a representative blot after short (upper panel) and long exposure (lower panel). Full blots are shown in Figure S2.

\subsection{Affinity Purification of Low-Abundant Proteins}

The expression levels of the target proteins in various experimental systems can vary significantly. In order to analyze if ALFA Selector ${ }^{\mathrm{CE}}$ can also capture low-abundant ALFA-tagged proteins, we applied $50 \mathrm{~mL}$ of a HeLa lysate containing only $100 \mathrm{nM}$ ALFAshGFP2 to $1 \mathrm{~mL}$ of ALFA Selector ${ }^{\mathrm{CE}}$ resin using gravity flow (flow rate, $\sim 1 \mathrm{~mL} / \mathrm{min}$ ). After washing, the column was eluted "in flow" with $1 \mathrm{mM}$ ALFA peptide in PBS. Under these experimental conditions, a nearly complete binding of the ALFA-tagged substrate to the 
resin was achieved (Figure 3C; Figure S2). Only after prolonged exposure, trace amounts of the target protein could be detected by Western blotting in the non-bound fraction. The affinity of ALFA Selector ${ }^{C E}$ is therefore sufficient for an efficient capture of ALFA-tagged proteins, even from dilute samples.

\subsection{Buffer Compatibility}

To compare the behavior of ALFA Selector ${ }^{\mathrm{PE}}$ and ALFA Selector ${ }^{\mathrm{CE}}$ in different buffers, both resins were first loaded with either shGFP2-ALFA or ALFA-shGFP2. After thorough washing with PBS, the resins were transferred into various buffers and analyzed after shaking for $2 \mathrm{~h}$ at room temperature (Figure 4). In general, both $\mathrm{N}$ - and C-terminally ALFA-tagged shGFP2 showed a remarkably stable association with both ALFA Selector resins, even in the presence of high salt concentrations (up to $>3 \mathrm{M} \mathrm{NaCl}$ ), nondenaturing detergents, and small-to-moderate amounts of urea. By contrast, denaturing detergents such as SDS and urea concentrations $>3 \mathrm{M}$ led to a substantial release of the target protein into the supernatant. For both resins, the substrate leakage was slightly more pronounced when using ALFA-shGFP2 as a substrate instead of shGFP2-ALFA (Figure 4, lower and upper panels). In line with the weaker interaction expected for the novel cold-elutable ALFA Selector ${ }^{\mathrm{CE}}$, this resin exhibited a higher tendency to release target proteins into the supernatant than ALFA Selector ${ }^{\mathrm{PE}}$ (compare Figure 4A,B).
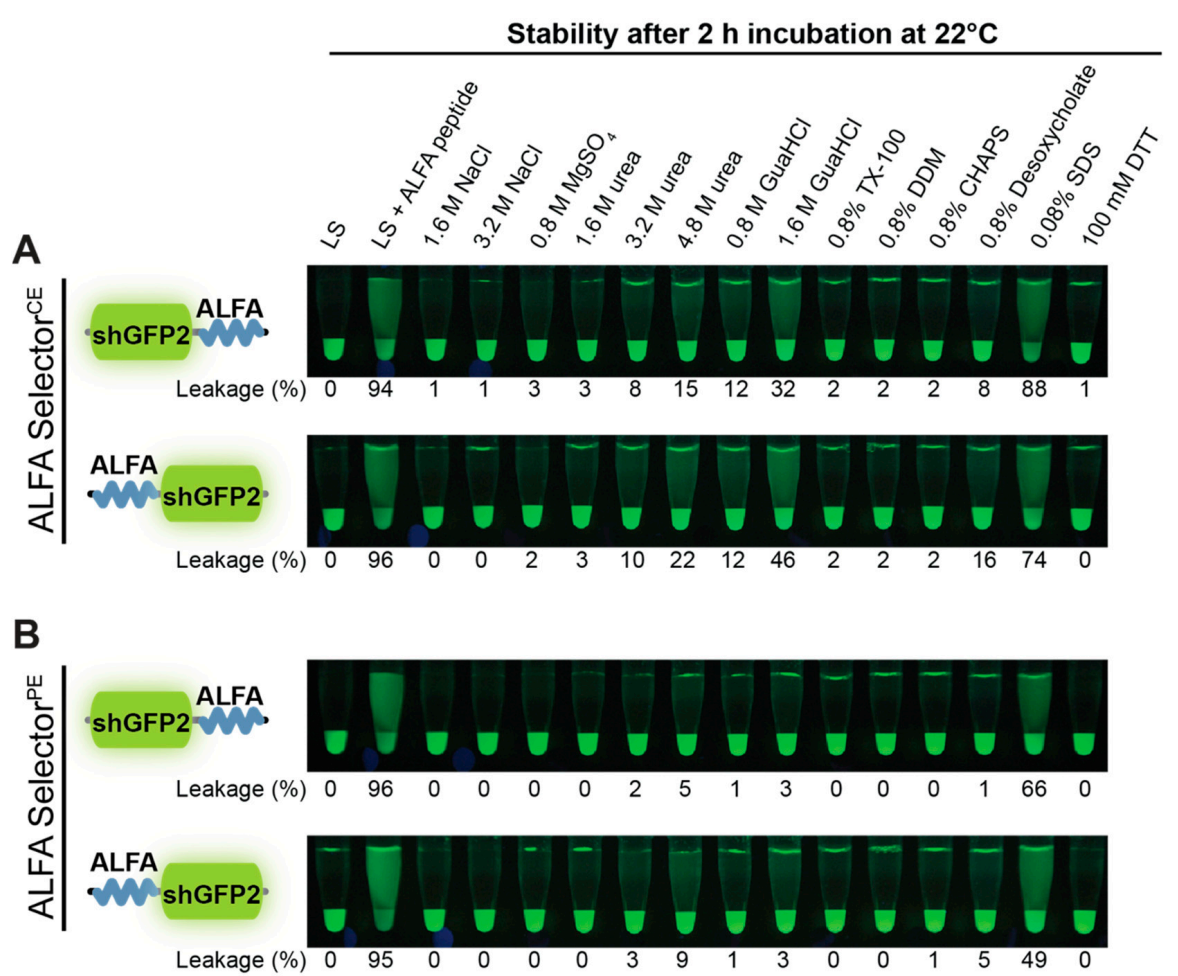

Figure 4. Buffer compatibility. ALFA Selector ${ }^{\mathrm{CE}}$ (A) and ALFA Selector ${ }^{\mathrm{PE}}$ (B) were saturated with shGFP2-ALFA (upper panels) or ALFA-shGFP2 (lower panels), washed extensively with PBS and incubated in a 10-fold volume of the indicated substances for $2 \mathrm{~h}$ at $22^{\circ} \mathrm{C}$. The leakage of target protein from the Selector resin was analyzed by quantifying the fluorescent material released into the supernatant before (shown here) and after post-elution with ALFA peptide (control shown in Figure S3). The degree of leakage/dissociation of target protein from the beads is given as $\%$ of total.

\subsection{Regeneration of ALFA Selector $C E$}

Finally, we analyzed if it was possible to regenerate ALFA Selector ${ }^{\mathrm{CE}}$ after use. Therefore, the resin was loaded with saturating amounts of ALFA-shGFP2, washed and eluted in flow mode with PBS containing $1 \mathrm{mM}$ ALFA peptide. After 1 and 10 cycles of regeneration under acidic or basic conditions (100 mM glycine, $\mathrm{pH}$ 2.2, and $150 \mathrm{mM} \mathrm{NaCl}$, or $100 \mathrm{mM}$ 
$\mathrm{NaOH}$, respectively), the same sequence of loading, washing and peptide elution was performed again. The absorption profiles (measured at $280 \mathrm{~nm}$ ) for the complete regeneration experiments performed under acidic (Figure 5A) and basic conditions (Figure S4A) showed only minor variations during the loading and elution of the ALFA-tagged target protein before regeneration and after 1 or 10 regeneration cycles. Similar amounts and quality of the eluted target protein were also observed when analyzing the respective eluates by SDS-PAGE and Coomassie staining (Figure 5B and Figure S4B), indicating that there is no obvious loss in binding capacity even after 10 regeneration cycles. In line with this observation, affinity purification experiments using the regenerated resins showed that the overall capacity and the low nonspecific background binding to ALFA Selector ${ }^{\mathrm{CE}}$ remained largely unchanged (Figure 5C and Figure S4C). ALFA Selector ${ }^{\mathrm{CE}}$ can therefore be used multiple times without a substantial loss of performance.
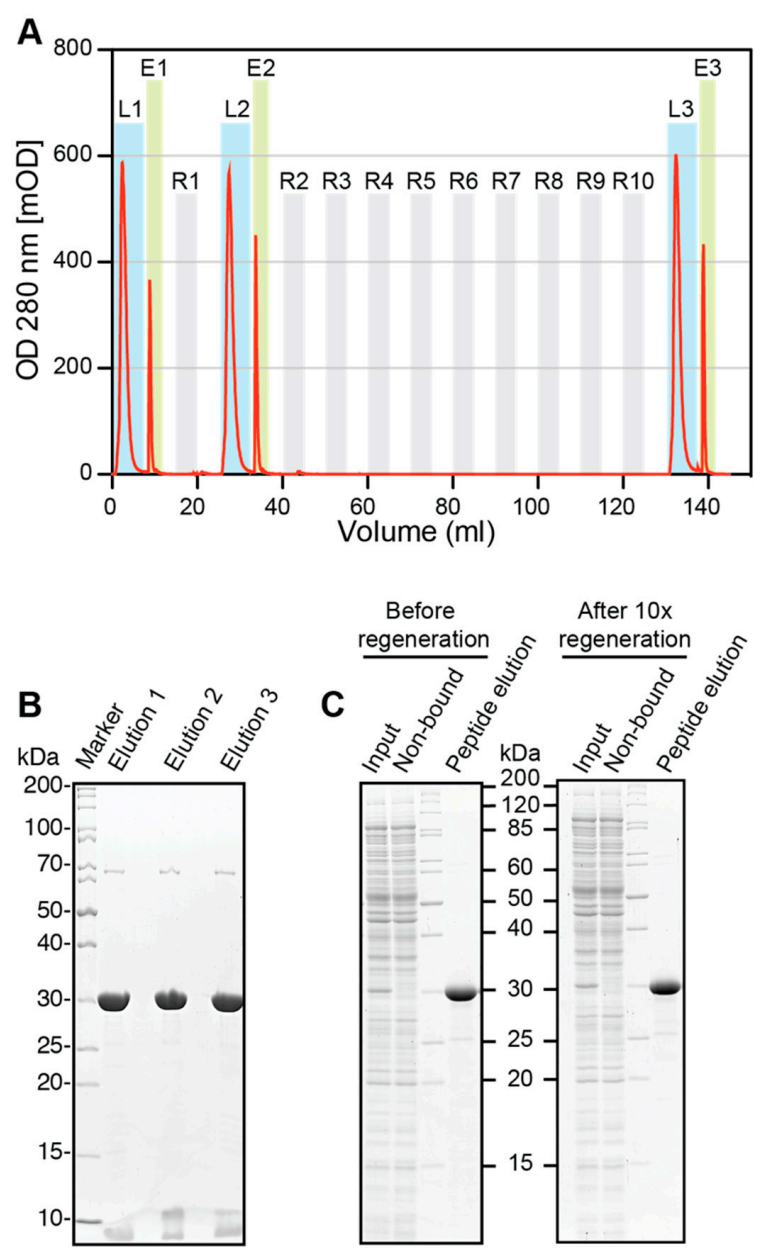

Figure 5. Regeneration of ALFA Selector ${ }^{C E}$ under acidic conditions. (A): $0.5 \mathrm{~mL}$ of ALFA Selector ${ }^{\mathrm{CE}}$ was subjected to a first cycle of loading with ALFA-shGFP2 (L1) and competitive peptide elution (E1). The column was regenerated under acidic conditions using $100 \mathrm{mM}$ glycine and $150 \mathrm{mM} \mathrm{NaCl}$ at $\mathrm{pH} 2.2$ (R1) and re-equilibrated with PBS before starting a second cycle of loading and elution (L2 and E2). After 9 additional repeated regeneration/re-equilibration steps (R2-R10), the column was loaded and eluted a third time (L3, E3). The whole procedure was followed by recording the optical density at $280 \mathrm{~nm}$ (OD280; red curves). (B): Fractions for each elution step were collected, quantified and analyzed by SDS-PAGE and Coomassie staining. (C): Effect of regeneration on nonspecific background binding. Single-step affinity purification from $E$. coli lysate blended with shGFP2-ALFA was performed using ALFA Selector ${ }^{\mathrm{CE}}$ either before regeneration or after 10 cycles of regeneration with glycine, $\mathrm{pH} 2.2$ (left and right panels, respectively). The experiment was essentially performed as described for Figure 3A,B. 


\section{Discussion}

We recently described the ALFA system comprising the ALFA-tag, a novel epitope tag and a set of ALFA-specific sdAbs, NbALFA and NbALFA ${ }^{\mathrm{PE}}$ [15]. NbALFA shows an extremely stable and robust association with ALFA-tagged proteins and is ideally suited for in vivo protein manipulations, highly sensitive affinity capturing and advanced detection and imaging techniques. By contrast, $\mathrm{NbALFA}{ }^{\mathrm{PE}}$ was optimized for competitive elution at ambient temperature, and can be used for the purification of proteins or demanding living cell isolation under physiological conditions [15]. To broaden the versatility of the ALFA system even further, we, here, aimed at developing $\mathrm{NbALFA}^{\mathrm{CE}}$, a new sdAb combining high selectivity and stable binding of ALFA-tagged proteins with an efficient competitive elution at $4{ }^{\circ} \mathrm{C}$ under physiological buffer conditions.

To find sdAbs fulfilling these virtually diverging goals, we started two independent phage-display screens based on different libraries. The first library was created by conventional immunization of alpacas, while the second library was designed by introducing mutations into $\mathrm{NbALFA}$ and $\mathrm{NbALFA}{ }^{\mathrm{PE}}$ by a rational, structure-guided approach. After applying stringent selection criteria, both libraries yielded $N b A L F A^{\mathrm{CE}}$ candidates that allowed stable binding of ALFA-tagged proteins while permitting an efficient competitive elution at $4{ }^{\circ} \mathrm{C}$. In addition to these requested features we could directly screen for, optimal $\mathrm{NbALFA}^{\mathrm{CE}}$ candidates should ideally show comparable binding strength and elution kinetics for target proteins harboring N- or C-terminal ALFA-tags.

Surprisingly, however, we noticed striking differences depending on the origin of the clones analyzed. Both $\mathrm{NbALFA}{ }^{\mathrm{CE}}$ candidates derived from the immunized library showed a strong preference for proteins harboring a C-terminal ALFA-tag. By contrast, clones originating from the semisynthetic library showed remarkably similar elution kinetics irrespective of the localization of the ALFA-tag within the target protein-just as described for $\mathrm{NbALFA}$ and $\mathrm{NbALFA}{ }^{\mathrm{PE}}$, indicating that the $\mathrm{NbALFA}^{\mathrm{CE}}$ candidates originating from the semisynthetic library preserved this feature.

The consistent recognition of target proteins tagged at either terminus is, therefore, not necessarily self-evident. By generating a semisynthetic library based on sdAbs that already possess this requested feature, we aimed to increase the chances of also finding these properties in the descendant clones. We believe that sdAb clones with similar properties may also be present in the immunized library. However, it is hard to envision a selection procedure that would result in sdAbs featuring these properties in a stringent and reproducible manner. Therefore, once a well-characterized sdAb displaying some of the desired features and biophysical properties is available, using this sdAb as the precursor of a new library seems to be an effective alternative to proceeding with a conventional de novo discovery process. In analogy, this general approach can potentially be applied to finetune certain binding properties (e.g., binding strength) while making sure the sdAb keeps other desired properties, such as its biochemical properties, its charge distribution or its specificity. Such an approach is most promising if a direct screening for a rare feature is not feasible or would require unreasonable effort.

The final NbALFA ${ }^{\mathrm{CE}}$ displays four modifications with respect to NbALFA. In comparison to the $\mathrm{NbALFA}^{\mathrm{PE}}$, featuring intermediate affinity, the off-rate of $\mathrm{NbALFA}^{\mathrm{CE}}$ is substantially enhanced, which is a prerequisite for achieving an efficient competitive elution at $4{ }^{\circ} \mathrm{C}$ (Figure 2E,F). The resin can, nevertheless, efficiently capture ALFA-tagged proteins even from dilute samples using a simple gravity-flow purification system (Figure 3C). As expected, the higher off-rate also influences the biochemical stability of the complexes formed with ALFA-tagged proteins. NbALFA ${ }^{\mathrm{CE}}$ is, therefore, slightly more sensitive than $\mathrm{NbALFA}^{\mathrm{PE}}$ towards denaturing reagents such as urea or guanidinium hydrochloride and certain detergents (Figure 4). However, under physiological buffer conditions or even at drastically elevated salt concentrations, any spontaneous leakage of target protein from an $\mathrm{NbALFA}^{\mathrm{CE}_{\text {-coupled resin }} \text { (ALFA Selector }}{ }^{\mathrm{CE}}$ ) is negligible (Figure 2E,F and Figure 4).

Although the N- and C-terminal ALFA-tagged proteins behaved in a strikingly similar way in the kinetic off-rate assays performed with ALFA Selector ${ }^{\mathrm{CE}}$, we observed subtle 
differences regarding their biochemical properties and pull-down behavior, especially under nonphysiological conditions (Figure 4).The observed differences in biochemical behavior are marginal for our model substrate shGFP2. However, as for any tag, it may be advisable to test the optimal position of the tag on other proteins or protein complexes, as steric effects or target-specific surface properties might influence its accessibility.

When varying the concentration of the ALFA peptide during elution from ALFA Selector ${ }^{\mathrm{CE}}$, we observed the sharpest elution profiles in stopped-flow and flow mode if $1 \mathrm{mM}$ peptide was used. At this concentration, the excess of peptide over resin-bound $\mathrm{sdAb}$ is sufficiently high to ensure a nearly off-rate-limited elution. Therefore, higher peptide concentrations will not increase the elution speed further. When using larger elution volumes, as generally performed in "batch" mode, peptide concentrations of only $200 \mu \mathrm{M}$ are fully sufficient (Figure 2), as a similar excess of peptide over the resin-bound $\mathrm{sdAb}$ is achieved. While competitive peptide elution at $4{ }^{\circ} \mathrm{C}$ from ALFA Selector ${ }^{\mathrm{CE}}$ requires 15-20 min for completion, elution at room temperature can even be performed using gravity flow (Figure 3C, Figure S1C and Figure 5).

At all tested temperatures, rapid and complete elution from ALFA Selector ${ }^{\mathrm{CE}}$ can also be achieved by applying acidic or basic conditions (Figure 5 and Figure S4), which was exploited to perform cleaning-in-place (CIP) regeneration. After 10 cycles of regeneration, the binding capacity and specificity of ALFA Selector ${ }^{\mathrm{CE}}$ remained largely unaffected (Figure $5 B, C$ and Figure S4B,C). ALFA Selector ${ }^{C E}$ can therefore be reused several times without a loss of performance.

In summary, by introducing the novel, cold-elutable $\mathrm{NbALFA}^{\mathrm{CE}}$, the same ALFAtagged protein can be bound by three alternative NbALFA variants featuring different affinities, thereby increasing the versatility of the ALFA system even further. ALFA Selector ${ }^{\mathrm{CE}}$ combines a highly specific and biochemically stable binding of ALFA-tagged proteins with the ability to readily elute at low temperatures under physiological buffer conditions. Thereby, the newly characterized ALFA Selector ${ }^{\mathrm{CE}}$ is an ideal tool for the clean, single-step purification of temperature-labile targets and applications requiring perfect structural and functional conservation, such as for cryo-electron microscopy or the purification of sensitive enzymes, respectively.

Supplementary Materials: The following are available online at https://www.mdpi.com/2218-273 X/11/2/269/s1. Figure S1: Titration of ALFA peptide concentration, Figure S2: One-step affinity purification of low abundant proteins, Figure S3: Buffer compatibility, Figure S4: Regeneration of ALFA Selector ${ }^{\mathrm{CE}}$ under basic conditions, Table S1: Primers used in this study, Table S2: Plasmids used in this study, Table S3: Antibodies and Selector resins used in this study.

Author Contributions: Conceptualization, M.K., H.G. and S.F.; methodology, M.K., H.G. and S.F.; validation, M.K., M.G.-B., A.C. and S.F.; formal analysis, M.K., H.G. and S.F.; investigation, M.K., H.G. and S.F.; resources, M.G.-B. and A.C.; data curation, M.K., H.G. and S.F.; writing-original draft preparation, M.K. and S.F.; writing-review and editing, M.K., H.G., M.G.-B., A.C., F.O. and S.F.; visualization, M.K., H.G. and S.F.; supervision, S.F.; project administration, S.F. All authors have read and agreed to the published version of the manuscript.

Funding: This research received no external funding.

Institutional Review Board Statement: All the work involving animal experiments was conducted in compliance with ethical regulations for animal research and testing. All the experiments conducted did not require ethical approval but were communicated to and accepted by the local authorities (LAVES Niedersachsen, Germany).

Conflicts of Interest: S.F., H.G. and F.O. are shareholders in NanoTag Biotechnologies GmbH. All the other authors declare no competing interests. 


\section{References}

1. Muyldermans, S. Nanobodies: Natural single-domain antibodies. Annu. Rev. Biochem. 2013, 82, 775-797. [CrossRef]

2. Lauwereys, M.; Ghahroudi, M.A.; Desmyter, A.; Kinne, J.; Hölzer, W.; De Genst, E.; Wyns, L.; Muyldermans, S.; Baral, T.N.; Retamozzo, V.C.; et al. Camelid immunoglobulins and nanobody technology. Vet. Immunol. Immunopathol. 2009, 128, 178-183. [CrossRef]

3. Revets, H.; De Baetselier, P.; Muyldermans, S. Nanobodies as novel agents for cancer therapy. Expert Opin. Biol. Ther. 2005, 5, 111-124. [CrossRef] [PubMed]

4. Ingram, J.R.; Schmidt, F.I.; Ploegh, H.L. Exploiting Nanobodies' Singular Traits. Annu. Rev. Immunol. 2018, 36, 695-715. [CrossRef] [PubMed]

5. Cheloha, R.W.; Harmand, T.J.; Wijne, C.; Schwartz, T.U.; Ploegh, H.L. Exploring cellular biochemistry with nanobodies. J. Biol Chem. 2020, 295, 15307-15327. [CrossRef] [PubMed]

6. Muruganandam, A.; Tanha, J.; Narang, S.; Stanimirovic, D. Selection of phage-displayed llama single-domain antibodies that transmigrate across human blood-brain barrier endothelium. FASEB J. 2002, 16, 240-242. [CrossRef] [PubMed]

7. Salema, V.; Fernández, L.Á. Escherichia coli surface display for the selection of nanobodies. Microb. Biotechnol. 2017, 10, 1468-1484. [CrossRef]

8. McMahon, C.; Baier, A.S.; Pascolutti, R.; Wegrecki, M.; Zheng, S.; Ong, J.X.; Erlandson, S.C.; Hilger, D.; Rasmussen, S.G.F.; Ring, A.M.; et al. Yeast surface display platform for rapid discovery of conformationally selective nanobodies. Nat. Struct. Mol. Biol. 2018, 25, 289-296. [CrossRef]

9. Moutel, S.; Bery, N.; Bernard, V.; Keller, L.; Lemesre, E.; De Marco, A.; Ligat, L.; Rain, J.C.; Favre, G.; Olichon, A.; et al. NaLi-H1: A universal synthetic library of humanized nanobodies providing highly functional antibodies and intrabodies. Elife 2016, 5, 1-31. [CrossRef]

10. Zimmermann, I.; Egloff, P.; Hutter, C.A.J.; Arnold, F.M.; Stohler, P.; Bocquet, N.; Hug, M.N.; Huber, S.; Siegrist, M.; Hetemann, L.; et al. Synthetic single domain antibodies for the conformational trapping of membrane proteins. Elife 2018, 7, 1-32. [CrossRef]

11. Jovčevska, I.; Muyldermans, S. The Therapeutic Potential of Nanobodies. BioDrugs 2020, 34, 11-26. [CrossRef] [PubMed]

12. Morrison, C. Nanobody approval gives domain antibodies a boost. Nat. Rev. Drug Discov. 2019, 18, 485-487. [CrossRef]

13. Schoof, M.; Faust, B.; Saunders, R.A.; Sangwan, S.; Rezelj, V.; Hoppe, N.; Boone, M.; Billesbølle, C.B.; Zimanyi, M.; Deshpande, I.; et al. An ultra-high affinity synthetic nanobody blocks SARS-CoV-2 infection by locking Spike into an inactive conformation. bioRxiv Prepr. Serv. Biol. 2020, 1-24. [CrossRef]

14. Esparza, T.J.; Martin, N.P.; Anderson, G.P.; Goldman, E.R.; Brody, D.L. High affinity nanobodies block SARS-CoV-2 spike receptor binding domain interaction with human angiotensin converting enzyme. Sci. Rep. 2020, 10, 1-13. [CrossRef]

15. Götzke, H.; Kilisch, M.; Martínez-Carranza, M.; Sograte-Idrissi, S.; Rajavel, A.; Schlichthaerle, T.; Engels, N.; Jungmann, R.; Stenmark, P.; Opazo, F.; et al. The ALFA-tag is a highly versatile tool for nanobody-based bioscience applications. Nat. Commun. 2019, 10, 1-12. [CrossRef] [PubMed]

16. Salzer, B.; Schueller, C.M.; Zajc, C.U.; Peters, T.; Schoeber, M.A.; Kovacic, B.; Buri, M.C.; Lobner, E.; Dushek, O.; Huppa, J.B.; et al. Engineering AvidCARs for combinatorial antigen recognition and reversible control of CAR function. Nat. Commun. 2020, 11, 1-16. [CrossRef]

17. Rudnick, S.I.; Adams, G.P. Affinity and avidity in antibody-based tumor targeting. Cancer Biother. Radiopharm. 2009, $24,155-161$. [CrossRef]

18. Fang, T.; Lu, X.; Berger, D.; Gmeiner, C.; Cho, J.; Schalek, R.; Ploegh, H.; Lichtman, J. Nanobody immunostaining for correlated light and electron microscopy with preservation of ultrastructure. Nat. Methods 2018, 15, 1029-1032. [CrossRef]

19. Virant, D.; Traenkle, B.; Maier, J.; Kaiser, P.D.; Bodenhöfer, M.; Schmees, C.; Vojnovic, I.; Pisak-Lukáts, B.; Endesfelder, U.; Rothbauer, U. A peptide tag-specific nanobody enables high-quality labeling for dSTORM imaging. Nat. Commun. 2018, 9, 1-14. [CrossRef] [PubMed]

20. Erreni, M.; Schorn, T.; D'autilia, F.; Doni, A. Nanobodies as versatile tool for multiscale imaging modalities. Biomolecules 2020, 10, 1695. [CrossRef]

21. Yang, Y.-S.S.; Moynihan, K.D.; Bekdemir, A.; Dichwalkar, T.M.; Noh, M.M.; Watson, N.; Melo, M.; Ingram, J.; Suh, H.; Ploegh, H.; et al. Targeting small molecule drugs to T cells with antibody-directed cell-penetrating gold nanoparticles. Biomater. Sci. 2018, 7 , 113-124. [CrossRef]

22. Kirchhofer, A.; Helma, J.; Schmidthals, K.; Frauer, C.; Cui, S.; Karcher, A.; Pellis, M.; Muyldermans, S.; Casas-Delucchi, C.S.; Cardoso, M.C.; et al. Modulation of protein properties in living cells using nanobodies. Nat. Struct. Mol. Biol. 2010, 17, 133-139. [CrossRef]

23. Rothbauer, U.; Zolghadr, K.; Tillib, S.; Nowak, D.; Schermelleh, L.; Gahl, A.; Backmann, N.; Conrath, K.; Muyldermans, S.; Cardoso, M.C.; et al. Targeting and tracing antigens in live cells with fluorescent nanobodies. Nat. Methods 2006, 3, 887-889. [CrossRef]

24. De Genst, E.J.; Guilliams, T.; Wellens, J.; Day, E.M.; Waudby, C.A.; Meehan, S.; Dumoulin, M.; Hsu, S.T.D.; Cremades, N.; Verschueren, K.H.G.; et al. Structure and properties of a complex of $\alpha$-synuclein and a single-domain camelid antibody. J. Mol. Biol. 2010, 402, 326-343. [CrossRef]

25. Brizzard, B. Epitope tagging. Biotechniques 2008, 44, 693-695. [CrossRef] [PubMed] 
26. Olichon, A.; De Marco, A. Preparation of a naïve library of camelid single domain antibodies. Methods Mol. Biol. 2012, 911, 65-78. [CrossRef]

27. Frey, S.; Görlich, D. A new set of highly efficient, tag-cleaving proteases for purifying recombinant proteins. J. Chromatogr. A 2014, 1337, 95-105. [CrossRef] [PubMed]

28. Frey, S.; Rees, R.; Schünemann, J.; Ng, S.C.; Fünfgeld, K.; Huyton, T.; Görlich, D. Surface Properties Determining Passage Rates of Proteins through Nuclear Pores. Cell 2018, 174, 202-217.e9. [CrossRef] 